\title{
An Optimization Design Strategy for Arithmetic Logic Unit
}

\author{
Jitesh R. Shinde ${ }^{1, *}$, Shilpa J. Shinde ${ }^{2}$ \\ ${ }^{1}$ Department of Electronics and Communication Engineering, Vaagdevi College of Engineering, India \\ ${ }^{2}$ M.Tech in Electronics Engineering, Nagpur, India
}

Copyright(C2019 by authors, all rights reserved. Authors agree that this article remains permanently open access under the terms of the Creative Commons Attribution License 4.0 International License

\begin{abstract}
The work in this paper presents a step by step optimization approach for the Arithmetic Logic Unit (ALU) at the logic circuit level. Herein concept of resource sharing (viz. operator sharing, functionality sharing), the concept of optimized arithmetic expressions (viz. arranging expression trees for minimum delay, sharing common subexpression, merging cascaded adders with carry) for optimization of combinational blocks in ALU had been used. The work in this paper shows how a simple tools like Deeds Digital Circuit Simulator (open source) or Aldec's Active HDL in combination with synthesis tool which can be used as effective teaching resource to teach concept of digital circuit design and thereby provides a vision to beginners how to start with VLSI project in VLSI digital domain and make it to a successful end.
\end{abstract}

Keywords Arithmetic Unit, Logical Unit, Arithmetic Logical Unit (ALU), Resource Sharing, Operator Sharing

\section{Introduction}

An arithmetic logic unit (ALU) is a combination of various digital circuits merged together to execute data processing instruction (i.e. arithmetic \& logical) in the central processing unit (CPU) of any processor or microcontroller or computer. ALU is basically a multi-function combinational digital logic circuit which requires one or two operands upon which it operates and produces the result.

The timing response of ALU depends on complexity \& manner in which the circuit is designed. There are various ways by which the circuit in HDL (Hardware Description Language) can be implemented. Some such ways are resource sharing \& optimized arithmetic expressions.

Resource sharing diminishes the amount of hardware required to implement HDL operations. Without resource sharing, each HDL operation is built with separate circuitry. For example, every ' + ' with noncomputable operands causes a new adder to be built. This repetition of hardware increases the area of design. In contrast, with resource sharing, several VHDL + operations can be implemented with a single adder to reduce the amount of hardware required. Also, different operations such as '+' and '-' can be assigned to a single adder or subtracter to reduce a design's circuit area further ${ }^{[11,12]}$. The resource sharing techniques used in the optimization of the ALU in this paper are operator sharing \& functionality sharing.

Operator sharing is a resource sharing technique to reduce the overall size of synthesized hardware. If the same operator is used in several different expressions, it can be shared. The sharing is done by routing the proper data to or from this particular operator via multiplexing circuits. Operators can be shared in mutually exclusive branches by proper routing of the input operands and/ or result. It is more beneficial for complex operators. The merit of sharing and the degree of saving depend on the relative complexity of the multiplexing circuit and the operator. However, sharing normally faces evaluation of the Boolean expressions and evaluations of the operators in cascade and this may introduce extra propagation delay.

Functionality sharing is a resource sharing technique. In a large, complex digital system, such as a processor, an array of functions is needed. Some functions or operations may be interlinked or may have some common functionality. If such common functions are implemented by a common circuit, then the approach is referred as functionality sharing. Example of functionality sharing can be an implementation of subtractor block using adder block using the concept of 2 's complement arithmetic ${ }^{[1,2]}$.

Optimized Arithmetic Expressions methodology uses the properties of arithmetic operators (such as commutative $\&$ associative properties of addition) to rearrange an expression so that it results in an optimized implementation. The three forms of arithmetic optimizations are arranging expression trees for minimum delay, merging cascaded adders with a carry, sharing common sub-expressions ${ }^{[1]}$.

ALU being a simple entity to understand for any beginner working in VLSI domain, hence it has selected as 
a case study in the work presented in this paper.

\section{ALU Design \& Implementation: Type I}

Before starting with any circuit design we have to think about the formal specification of the circuit. In other words, how a general or traditionally design ALU in CPU blocks works, what functions or instructions it executes and how many inputs and outputs it should have \& what basic building blocks are there in the circuit to execute the given instructions ${ }^{[2]}$. The basics of ALU design are available in the literature mentioned in the references ${ }^{[3,4,5,6,7 \& 8]}$.

Basically, an ALU should be capable of executing following arithmetic \& logical functions as listed in tables $2.1 \& 2.2$.

The tables 2.2 suggests that one logical block having some logical gates (four) with 4:1 multiplexer will be required to implement logical operations AND, OR, XOR and NOT in ALU to get an output of logical block.

The table 1.2 suggests that one arithmetic block consisting of 8 instances of one-bit full adder block with two 8:1 multiplexer will be required to implement operations Addition, Subtraction, Increment, Decrement and Passing of Input signal and thereby getting an output of arithmetic block. The input logic for carry-in 'Cin' \& 'B' input terminal of each full adder had been decided as per table (2.2). Depending on the input combination available on the select lines (i.e. S2, S1 \& S0) of 8:1 multiplexer, only selected full adder output viz. sum \& carry-out will be available at the output of multiplexer. For example, if $\mathrm{S} 2 \mathrm{~S} 1 \mathrm{~S} 0 \mathrm{C}$ in $=$ "0000", then ALU will execute transfer " $\mathrm{A}$ " operation (table 1.2). Herein first full adder output (leftmost full adder block) will be available at the output ' $\mathrm{Y}$ ' (figure 2.1). For $A={ }^{\prime} 0$ ' then output ' $Y$ ' $=$ ' 0 ' and for $A={ }^{\prime} 1$ ' then output ' $\mathrm{Y}$ ' = ' 1 .'

In addition to this, one 2:1 multiplexer will also be required to decide at given instance of time whether ALU is implementing arithmetic or logical function. If select bit ' $\mathrm{Si}$ ' (S3 in figure 2.1) of multiplexer equals to' 1 ', then ALU will perform logical operation otherwise it will perform arithmetic operations.

Thus, combinational digital blocks to implement an ALU in traditional approach are two 8:1 multiplexer, one 2:1 multiplexer, 8 one-bit full adder block \& 4 two-input logic gates, two NOT gates along with some constants (logic high or logic low) to get final output ' $\mathrm{Y}$ ' of one bit ALU.

The logical circuit diagram was implemented in Deeds circuit simulator \& using Aldec's Active HDL tool with Altera's (Intel) Quartus synthesis tool.

Table 2.1. Listing logical operations performed by ALU

\begin{tabular}{|c|c|c|c|c|}
\hline \multicolumn{3}{|c|}{ Function Select } & Output & \multirow{2}{*}{ Operation } \\
\hline S1 & S0 & Cin & $\mathrm{Y}$ & \\
\hline 0 & 0 & $\mathrm{X}$ & $\mathrm{Y}=\mathrm{A}$ OR B & OR \\
\hline 0 & 1 & $\mathrm{X}$ & $\mathrm{Y}=\mathrm{A} A \mathrm{AND} \mathrm{B}$ & AND \\
\hline 1 & 0 & $\mathrm{X}$ & $\mathrm{Y}=\mathrm{A} \mathrm{XOR} \mathrm{B}$ & XOR \\
\hline 1 & 1 & $\mathrm{X}$ & $\mathrm{Y}=\mathrm{A}^{\prime}$ & NOT \\
\hline \multicolumn{5}{|c|}{ Note $: A^{\prime}$ indicates $A b a r \&$ Cin $=X$ i.e. don't care } \\
\hline
\end{tabular}

Table 2.2. Listing arithmetic functions performed by ALU

\begin{tabular}{|c|c|c|c|c|c|c|}
\hline \multicolumn{3}{|c|}{ Function Select } & \multicolumn{2}{|c|}{ Inputs to Adder } & \multirow{2}{*}{$\begin{array}{c}\text { Output } \\
\mathrm{Y} \\
\end{array}$} & \multirow{2}{*}{ Operation } \\
\hline S2 & S1 & S0 & Cin & $\mathrm{B}$ & & \\
\hline 0 & 0 & 0 & 0 & 0 & $\mathrm{Y}=\mathrm{A}$ & Transfer A \\
\hline 0 & 0 & 1 & 1 & 0 & $\mathrm{Y}=\mathrm{A}+1$ & Increment A \\
\hline 0 & 1 & 0 & 0 & B & $\mathrm{Y}=\mathrm{A}+\mathrm{B}$ & Add $\mathrm{B}$ to $\mathrm{A}$ \\
\hline 0 & 1 & 1 & 1 & $\mathrm{~B}$ & $\mathrm{Y}=\mathrm{A}+\mathrm{B}+1$ & Add B to A plus 1 \\
\hline 1 & 0 & 0 & 0 & $\mathrm{~B}^{\prime}$ & $\mathrm{Y}=\mathrm{A}+\mathrm{B}^{\prime}$ & Add 1's complement of B to A \\
\hline 1 & 0 & 1 & 1 & B' & $\mathrm{Y}=\mathrm{A}+\mathrm{B}^{\prime}+1$ & Add 2's complement of B to A \\
\hline 1 & 1 & 0 & 0 & 1 & $\mathrm{Y}=\mathrm{A}-1=\mathrm{A}^{\prime}$ & Decrement A \\
\hline 1 & 1 & 1 & 1 & 1 & $\mathrm{Y}=\mathrm{A}$ & Transfer A \\
\hline
\end{tabular}




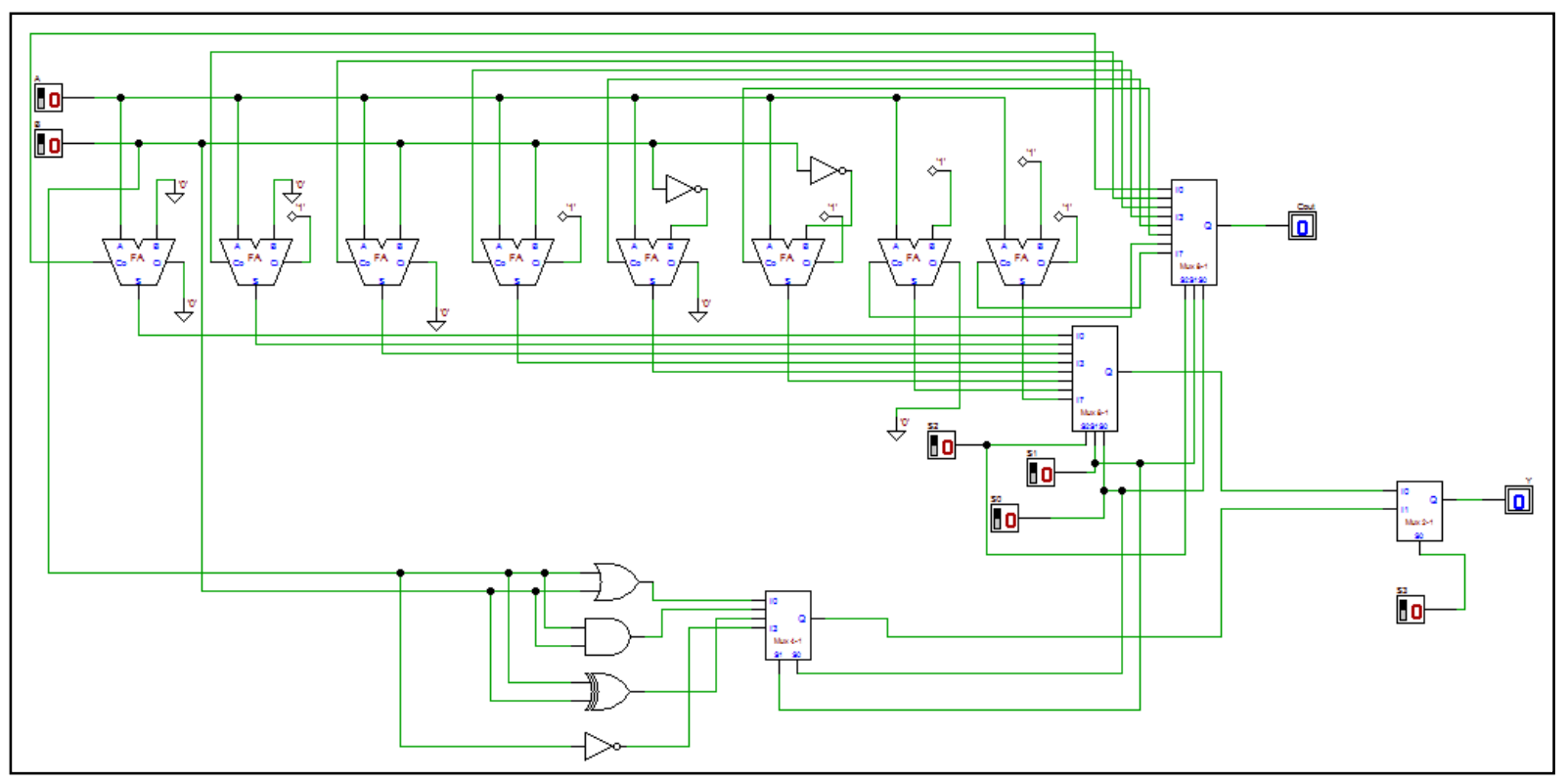

Figure 2.1. Circuit schematic of 1-bit ALU (Deeds DCS)

\begin{tabular}{|ll|}
\hline Flow Status & Successful - \\
Quartus II Version & 11.0 Build 208 07/03/2011 SP 1 SJ Web Edition \\
Revision Name & alugen \\
Top-level Entity Name & alugen \\
Family & Cyclone III \\
Device & EP3C16F484C6 \\
Timing Models & Final \\
Total logic elements & $5 / 15,408(<1 \%)$ \\
$\quad$ Total combinational functions & $5 / 15,408(<1 \%)$ \\
$\quad$ Dedicated logic registers & $0 / 15,408(0 \%)$ \\
Total registers & 0 \\
Total pins & $50 / 347(14 \%)$ \\
Total virtual pins & 0 \\
Total memory bits & $0 / 516,096(0 \%)$ \\
Embedded Multiplier 9-bit elements & $0 / 112(0 \%)$ \\
Total PLLs: & $0 / 4(0 \%)$
\end{tabular}

Figure 2.2. Flow summary of entity 'alugen' 


\begin{tabular}{|c|c|c|c|c|c|c|}
\hline \multicolumn{7}{|c|}{ Propagation Delay } \\
\hline & Input Port & Output Port & $\mathrm{RR}$ & RF & FR & FF \\
\hline 1 & A & oCout & 4.699 & & & 5.510 \\
\hline 2 & iA & oY & 4.639 & 4.635 & 5.405 & 5.401 \\
\hline 3 & iB & oCout & 4.996 & 5.073 & 5.788 & 5.881 \\
\hline 4 & iB & oY & 4.661 & 4.657 & 5.438 & 5.434 \\
\hline 5 & iso & oCout & 4.885 & & & 5.728 \\
\hline 6 & iso & oY & 4.653 & 4.649 & 5.454 & 5.450 \\
\hline 7 & is1 & oCout & 5.202 & & & 6.120 \\
\hline 8 & is 1 & oY & 4.506 & 4.526 & 5.348 & 5.349 \\
\hline 9 & is2 & oCout & 4.924 & & & 5.780 \\
\hline 10 & is2 & oY & 4.571 & 4.567 & 5.351 & 5.347 \\
\hline 11 & is3 & oY & 4.459 & 4.455 & 5.231 & 5.231 \\
\hline
\end{tabular}

Figure 2.3. Propagation delay summary of entity 'alugen' (delay in nsec)

\begin{tabular}{|c|c|}
\hline PowerPlay Power Analyzer Status & Successful - \\
\hline Quartus II Version & 11.0 Build 208 07/03/2011 SP 1 SJ Web Edition \\
\hline Revision Name & alugen \\
\hline Top-level Entity Name & alugen \\
\hline Family & Cyclone III \\
\hline Device & $\mathrm{EP} 3 \mathrm{C} 16 \mathrm{~F} 484 \mathrm{C} 6$ \\
\hline Power Models & Final \\
\hline Total Thermal Power Dissipation & $105.42 \mathrm{~mW}$ \\
\hline Core Dynamic Thermal Power Dissipation & $0.76 \mathrm{~mW}$ \\
\hline Core Static Thermal Power Dissipation & $51.81 \mathrm{~mW}$ \\
\hline I/O Thermal Power Dissipation & $52.85 \mathrm{~mW}$ \\
\hline Power Estimation Confidence & Low: user provided insufficient toggle rate data \\
\hline
\end{tabular}

Figure 2.4. Power consumption of entity 'alugen'

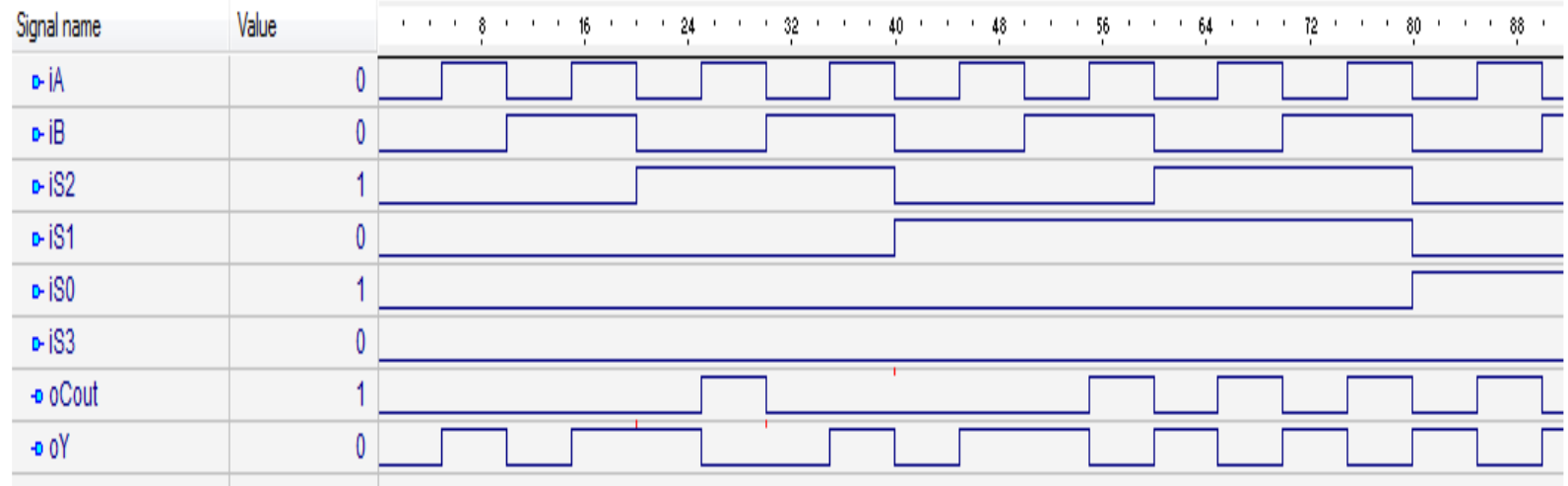

Figure 2.5. Output waveform of entity 'alugen' 


\section{ALU Design \& Implementation: Type II}

Herein this approach, the concept of resource \& operator sharing had been used to optimize the ALU. Herein, only one full adder block had been used to implement arithmetic operations given in table 2.1. The input logic for input terminal carry-in of full adder (as per table 2.2) is decided by 8:1 multiplexer i.e. for example: for $\mathrm{S} 2 \mathrm{~S} 1 \mathrm{~S} 0=$ " $000 "$, carry-in of full adder block is connected to logic ' 0 '. Similarly, the input logic for input terminal ' $B$ ' of full adder (as per table 2.1) is decided by 8:1 multiplexer i.e. for example: for $\mathrm{S} 2 \mathrm{~S} 1 \mathrm{~S} 0=$ " 000 ", input terminal 'B' of full adder block is connected to logic ' 0 '. Rest of the working of the circuit is same as discussed in previous section.

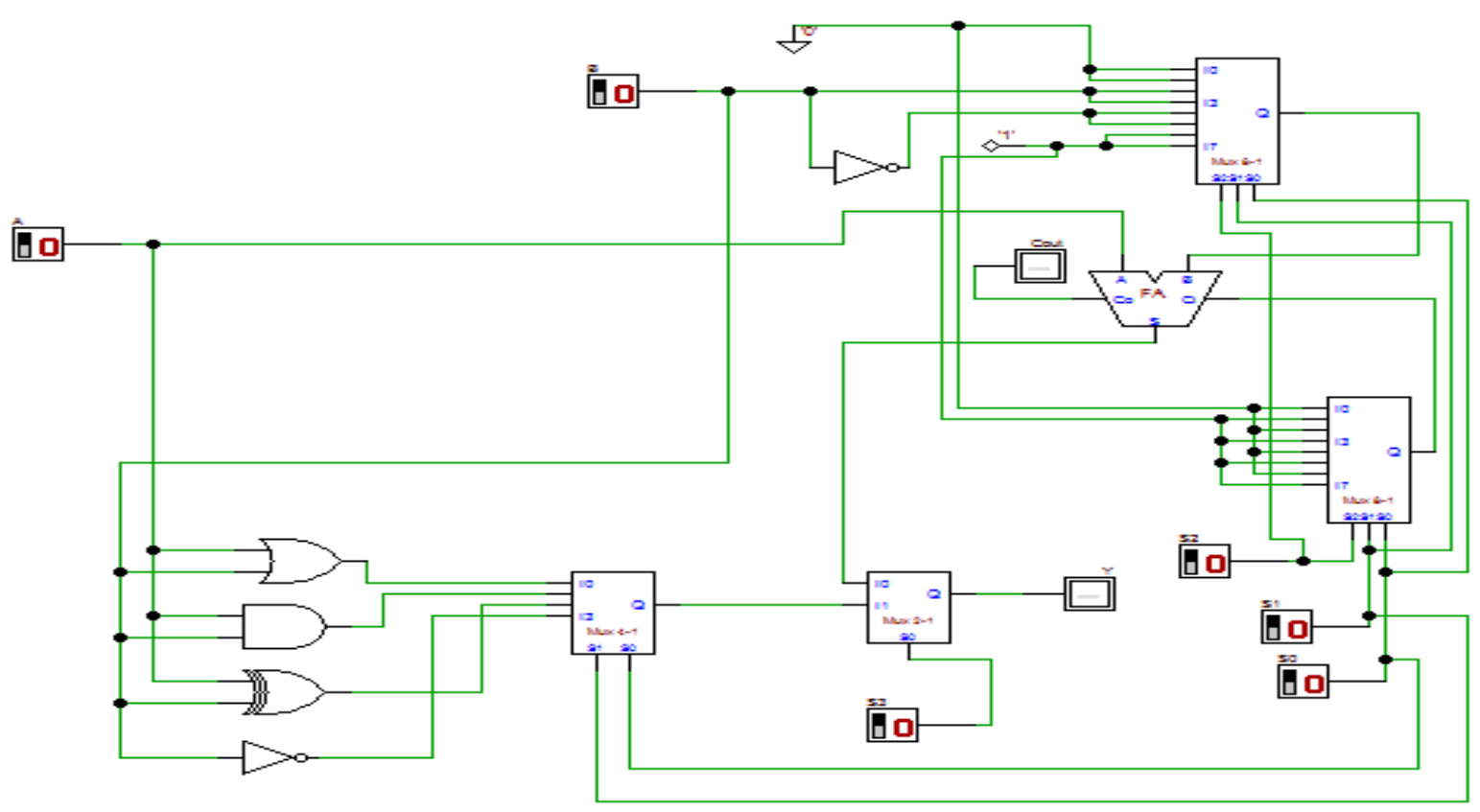

Figure 3.1. Circuit schematic of entity 'alugenopt'

\begin{tabular}{|c|c|}
\hline Flow Status & Successful - \\
\hline Quartus II Version & 11.0 Build 208 07/03/2011 SP 1 SJ Web Edition \\
\hline Revision Name & alugenopt \\
\hline Top-level Entity Name & alugenopt \\
\hline Family & Cyclone III \\
\hline Device & $\mathrm{EP} 3 \mathrm{C} 16 \mathrm{~F} 484 \mathrm{C} 6$ \\
\hline Timing Models & Final \\
\hline Total logic elements & $5 / 15,408(<1 \%)$ \\
\hline Total combinational functions & $5 / 15,408(<1 \%)$ \\
\hline Dedicated logic registers & $0 / 15,408(0 \%)$ \\
\hline Total registers & 0 \\
\hline Total pins & $50 / 347(14 \%)$ \\
\hline Total virtual pins & 0 \\
\hline Total memory bits & $0 / 516,096(0 \%)$ \\
\hline Embedded Multiplier 9-bit elements & $0 / 112(0 \%)$ \\
\hline Total PLLs & $0 / 4(0 \%)$ \\
\hline
\end{tabular}

Figure 3.2. Flow summary of entity 'alugenopt' 


\begin{tabular}{|c|c|c|c|c|c|c|}
\hline \multicolumn{7}{|c|}{ Propagation Delay } \\
\hline & Input Port & Output Port & RR & RF & $\mathrm{FR}$ & FF \\
\hline 1 & iA & oCout & 4.299 & & & 5.071 \\
\hline 2 & iA & oY & 4.839 & 4.895 & 5.615 & 5.664 \\
\hline 3 & iB & oCout & 4.591 & 4.610 & 5.360 & 5.434 \\
\hline 4 & iB & oY & 5.143 & 5.202 & 5.967 & 6.026 \\
\hline 5 & iso & oCout & 4.484 & & & 5.286 \\
\hline 6 & iso & oY & 5.024 & 5.077 & 5.807 & 5.878 \\
\hline 7 & is $1 \longdiv { \text { iso } }$ & oCout & 4.803 & & & 5.681 \\
\hline 8 & iS1 & oY & 5.351 & 5.401 & 6.214 & 6.273 \\
\hline 9 & iS2 & oCout & 4.444 & & & 5.241 \\
\hline 10 & is2 & oY & 4.992 & 5.042 & 5.774 & 5.833 \\
\hline 11 & iS3 & oY & 4.505 & 4.906 & 5.625 & 5.286 \\
\hline
\end{tabular}

Figure 3.3. Propagation delay summary of entity 'alugenopt' (delay in nsec)

\begin{tabular}{ll}
\hline \hline PowerPlay Power Analyzer Status & Successful - \\
Quartus II Version & 11.0 Build 208 07/03/2011 SP 1SJ Web Edition \\
Revision Name & alugenopt \\
Top-level Entity Name & alugenopt \\
Family & Cyclone III \\
Device & EP3C16F484C6 \\
Power Models & Final \\
Total Thermal Power Dissipation & $101.46 \mathrm{~mW}$ \\
Core Dynamic Thermal Power Dissipation & $0.63 \mathrm{~mW}$ \\
Core Static Thermal Power Dissipation & $51.80 \mathrm{~mW}$ \\
I/O Thermal Power Dissipation & $49.04 \mathrm{~mW}$ \\
Power Estimation Confidence & Low: user provided insufficient toggle rate data
\end{tabular}

Figure 3.4. Power consumption summary of entity 'alugenopt'

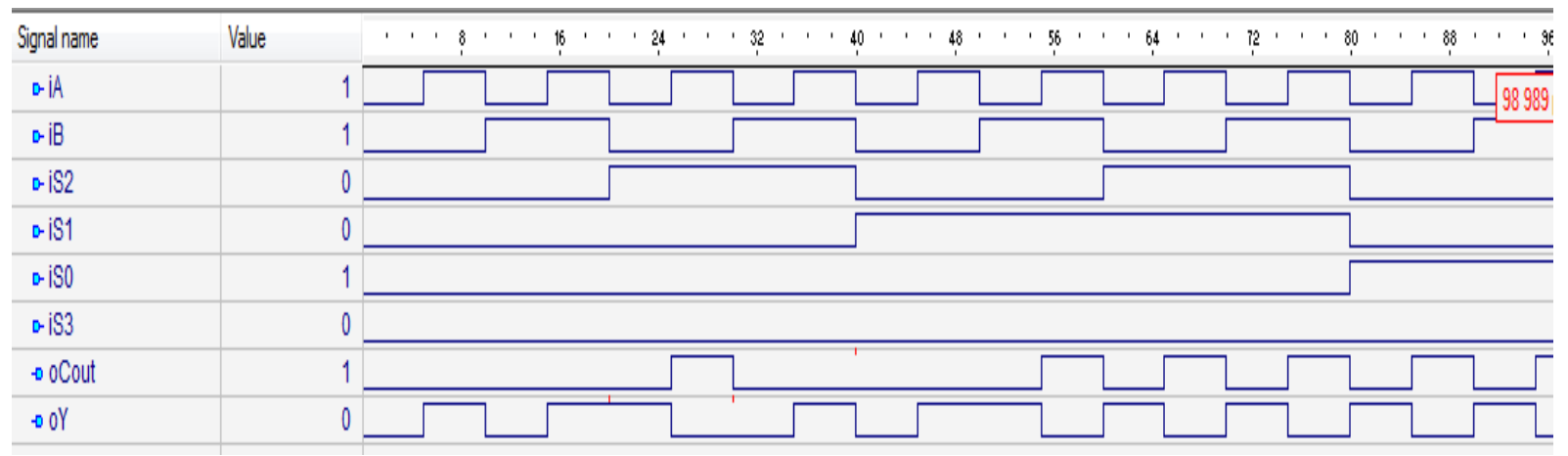

Figure 3.5. Output waveform of entity 'alugenopt'

\section{ALU Design \& Implementation: Type III}

The general ALU architecture that can be inferred from section II is given in figure 4.1.

The strategy used in type III ALU optimization is based on the concept on resource sharing and concept of optimized arithmetic expressions.

In type III strategy, all arithmetic, as well as logical instructions are realized using one full adder block only.
The approach used in the realization of the final optimized logic circuit shown in figure 4.6 is illustrated with the help of figures from 4.1 to 4.5 and tables from 4.1 to 4.6 respectively.

\section{Arithmetic Unit Realization}

In table 4.1, input literal ' $\mathrm{X}$ ' of full adder block had been kept fixed. Then, depending on various operations that an arithmetic block needs to execute, input literal ' $\mathrm{Y}$ ' is 
decided. Finally, SOP (sum of product) logical equation for literal ' $\mathrm{Y}$ ' in an optimized way is realized using 2:1 multiplexer (figure 4.4). The process is illustrated diagrammatically in figure $4.2,4.3$ and 4.4 respectively.

\section{Logical Unit Realization}

To find the equation for input literal ' $\mathrm{X}$ ' of full adder block, first, the logic equation for literal ' $\mathrm{X}$ ' for each gate is computed using the concept of K-map wherein SOP (sum of product) equation for literal ' $\mathrm{X}$ ' has been computed as a function of literal viz. 'A' \& 'B' respectively. This process had been illustrated in tables from 4.3 to 4.6 respectively and then table 4.2 had been realized. Accordingly, the logical unit shown in figure 4.5 had been realized. The concept of K-map here used to find the logical equation for literal ' $\mathrm{X}$ ' for corresponding gates \& then final SOP (sum of product) equation for ' $\mathrm{X}$ ' as shown in figure 4.5.

The final block diagram of an optimized 1-bit ALU is shown in figure 4.6.

The logical circuit diagram implemented in Deeds DCS circuit simulator \& waveform, flow summary, propagation delay report \& power consumption analysis report obtained from Altera's (Intel) Quartus tool are shown in figure 4.7 to 4.11 respectively.

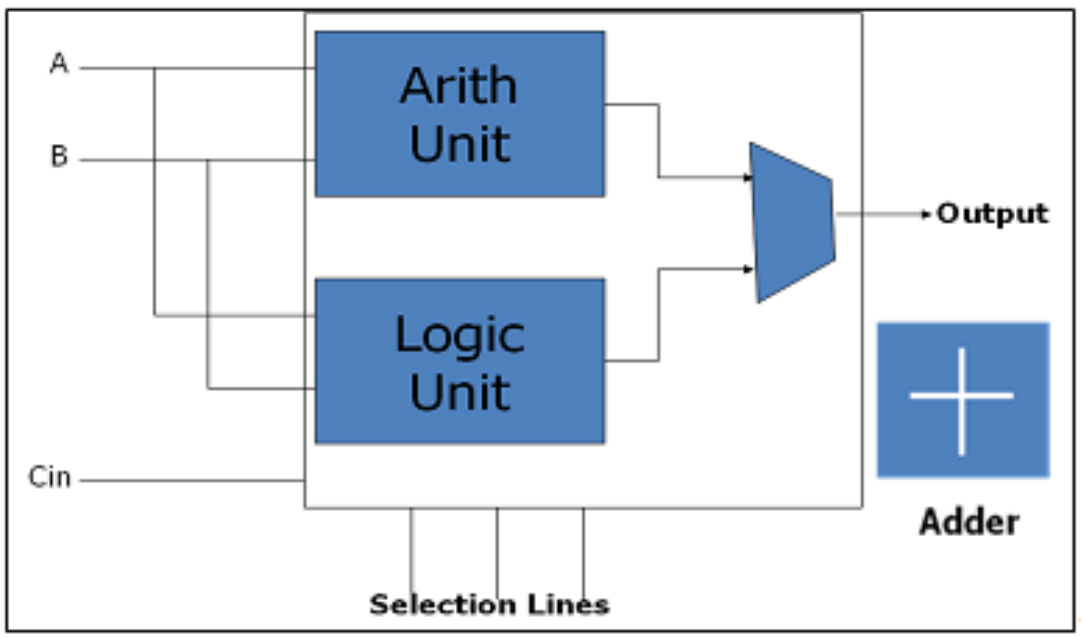

Figure 4.1. Block diagram of 1 bit ALU

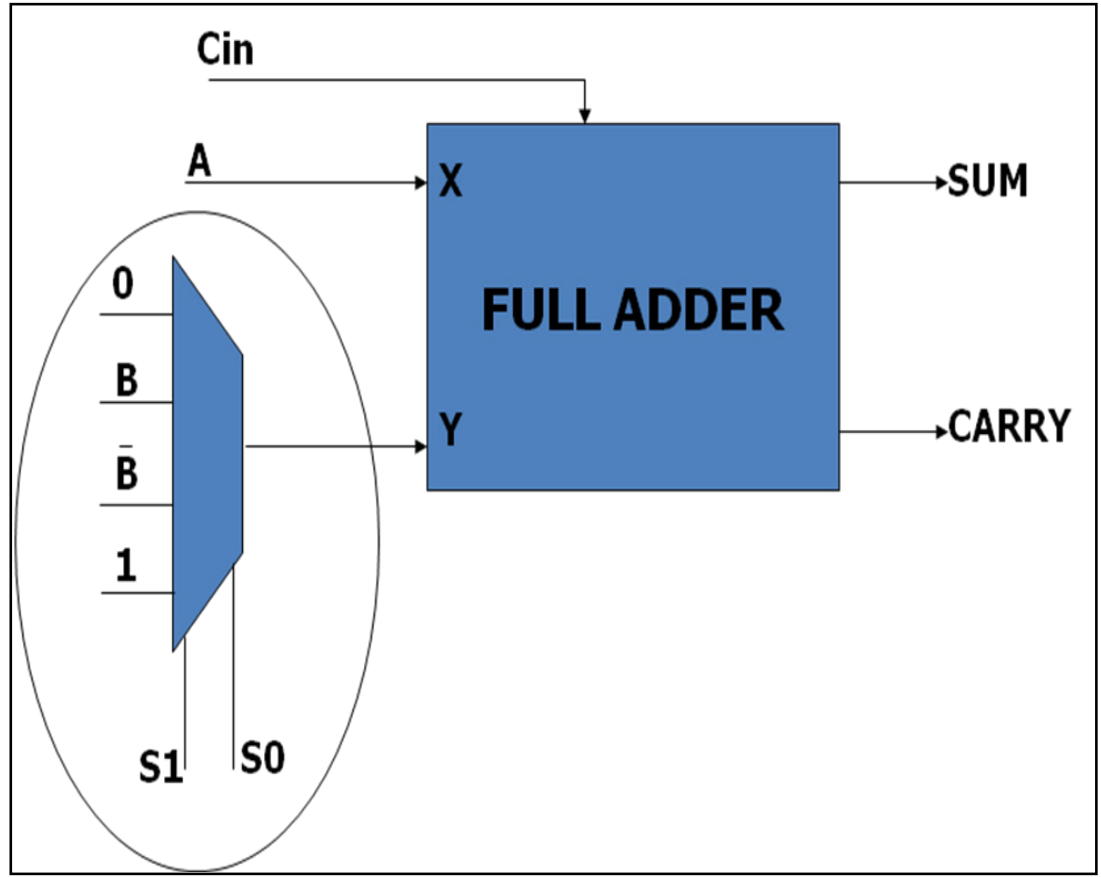

Figure 4.2. Arithmetic unit logic in an optimized 1-bit ALU using 4:1 multiplexer 


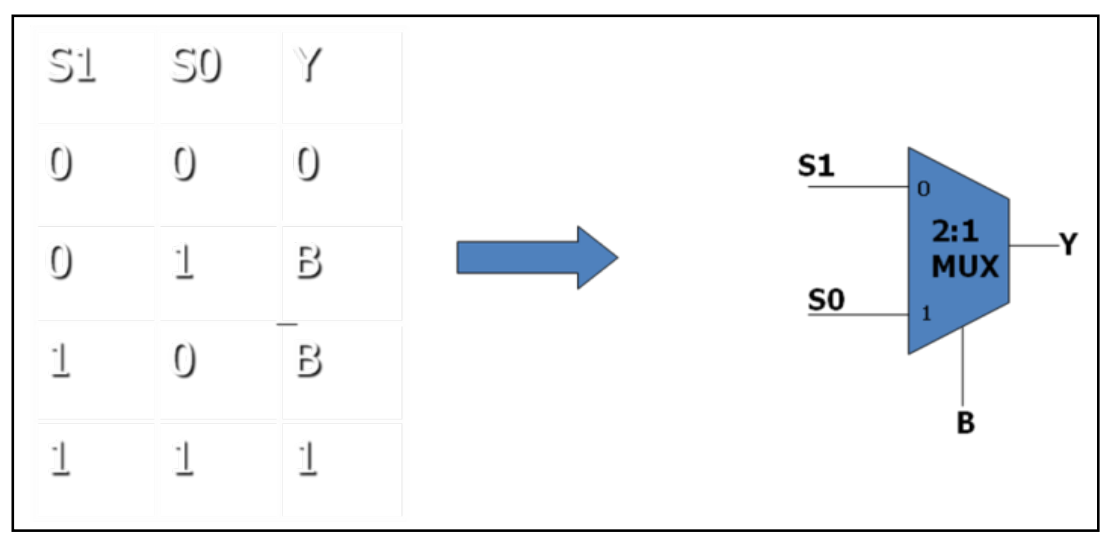

Figure 4.3. Arithmetic unit logic in an optimized 1-bit ALU using 2:1 multiplexer

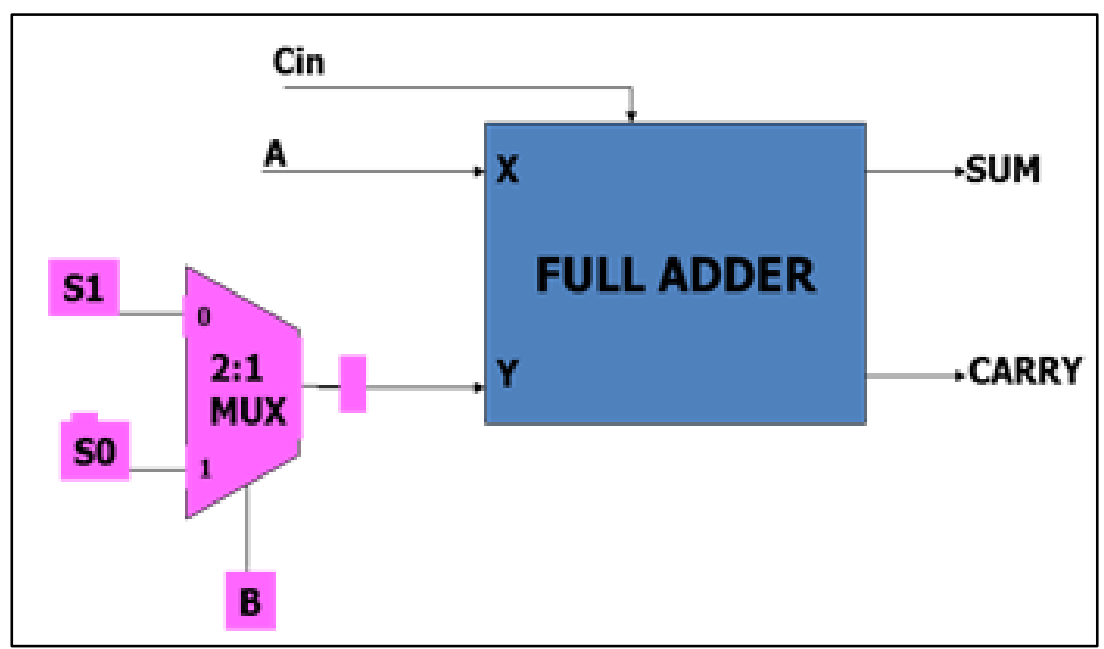

Figure 4.4. Arithmetic unit logic in an optimized 1-bit ALU using 2:1 multiplexer

Table 4.1. Truth Table for Arithmetic Unit for an optimized 1-bit ALU

\begin{tabular}{|c|c|c|c|c|c|c|}
\hline S2 & S1 & S0 & Cin & X & Y & Operation \\
\hline 0 & 0 & 0 & 0 & A & 0 & Transfer A \\
\hline 0 & 0 & 0 & 1 & A & 0 & Increment A \\
\hline 0 & 0 & 1 & 0 & A & B & Add B to A \\
\hline 0 & 0 & 1 & 1 & A & B & Add B to A plus 1 \\
\hline 0 & 1 & 0 & 0 & A & B' & Add 1's complement of B to A \\
\hline 0 & 1 & 0 & 1 & A & B' & Decrement A \\
\hline 0 & 1 & 1 & 0 & A & 1 & Transfer A \\
\hline 0 & 1 & 1 & 1 & A & 1 & A \\
\hline
\end{tabular}

Table 4.2. Truth Table for Logical Unit

\begin{tabular}{|c|c|c|c|c|c|c|}
\hline S2 & S1 & S0 & Cin & X & Y & Operation \\
\hline 1 & 0 & 0 & $\mathrm{x}$ & A+B & 0 & OR \\
\hline 1 & 0 & 1 & $\mathrm{x}$ & $\mathrm{A}^{\prime} \mathrm{B}$ & $\mathrm{B}$ & XND \\
\hline 1 & 1 & 0 & $\mathrm{x}$ & $\mathrm{A}^{\prime}$ & $\mathrm{B}^{\prime}$ & NOT \\
\hline 1 & 1 & 1 & $\mathrm{x}$ & $\mathrm{B}$ & 1 & NOR \\
\hline
\end{tabular}


Table 4.3. Truth Table for finding input ' $X$ ' for ORing operation i.e. $X(A, B)=A+B$

\begin{tabular}{|l|l|l|c|c|}
\hline A & B & X & Y=0 & 0 \\
\hline 0 & 0 & 0 & 0 & 0 \\
\hline 0 & 1 & 1 & 0 & 1 \\
\hline 1 & 0 & 1 & 0 & 1 \\
\hline
\end{tabular}

Table 4.4. Truth Table to find input ' $X$ ' for ANDing operation i.e. $X(A, B)=A$ ' $B$

\begin{tabular}{|c|c|c|c|c|}
\hline A & B & X & Y=B & 0 \\
\hline 0 & 0 & 0 & 1 & 0 \\
\hline 0 & 1 & 1 & 0 & 0 \\
\hline 1 & 0 & 0 & 1 & 1 \\
\hline
\end{tabular}

Table 4.5. Truth Table to find input ' $X$ ' for $X O R i n g$ operation i.e. $X(A, B)=A$ '

\begin{tabular}{|l|l|l|c|c|}
\hline A & B & X & Y=B' & 1 \\
\hline 0 & 0 & 1 & 0 & 0 \\
\hline 0 & 1 & 1 & 1 & 1 \\
\hline 1 & 0 & 0 & 0 & 0 \\
\hline
\end{tabular}

Table 4.6. Truth Table to find input ' $\mathrm{X}$ ' for NOT operation i.e. $\mathrm{X}(\mathrm{A}, \mathrm{B})=\mathrm{B}$

\begin{tabular}{|c|c|c|c|c|}
\hline A & B & X & Y=1 & Operation \\
\hline 0 & 0 & 0 & 1 & 1 \\
\hline 0 & 1 & 1 & 1 & 0 \\
\hline 1 & 0 & 0 & 1 & 1 \\
\hline 1 & 1 & 1 & 1 & 0 \\
\hline
\end{tabular}

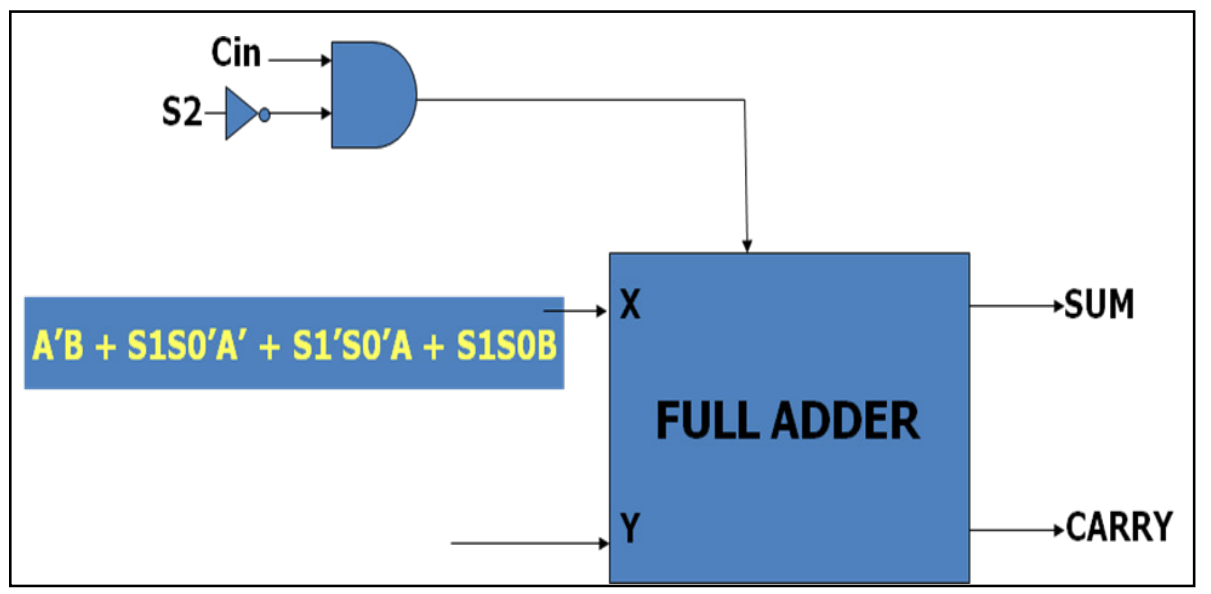

Figure 4.5. An optimized logical block for 1-bit ALU 


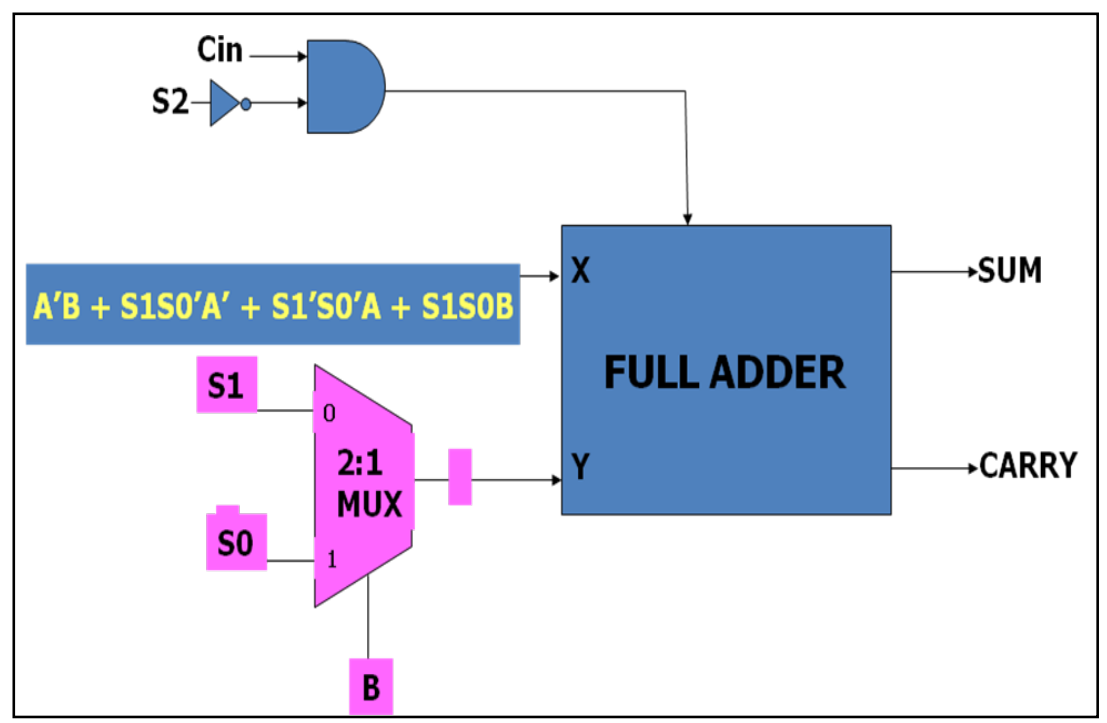

Figure 4.6. An optimized 1-bit ALU

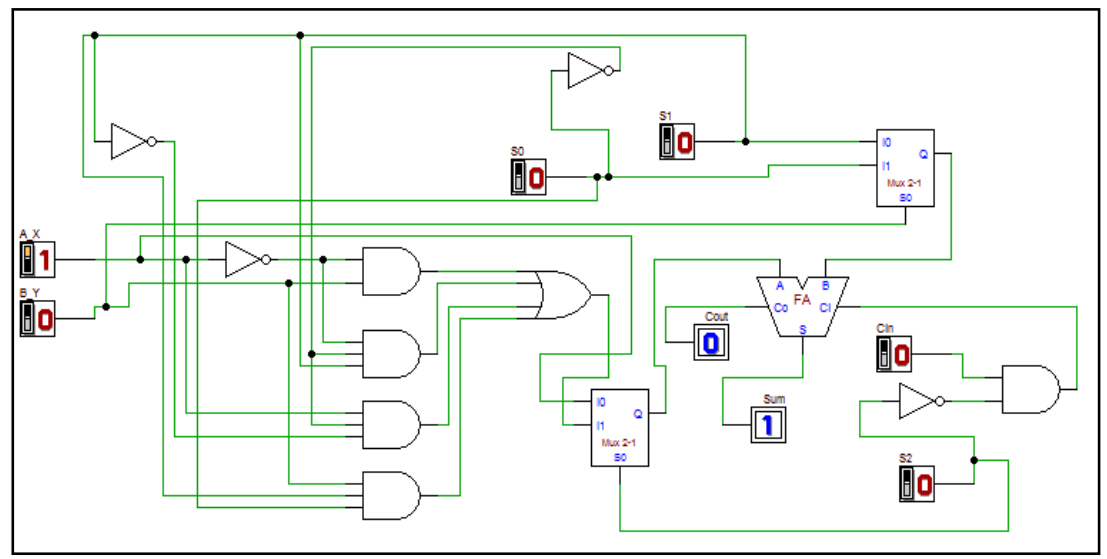

Figure 4.7. Circuit schematic of 1 bit optimized ALU 'aluopt'

\begin{tabular}{|ll|}
\hline Flow Status & Successful \\
Quartus II Version & 11.0 Build 208 07/03/2011 SP 1 SJ Web Edition \\
Revision Name & aluopt \\
Top-level Entity Name & aluopt \\
Family & Cyclone III \\
Device & EP3C16F484C6 \\
Timing Models & Final \\
Total logic elements & $5 / 15,408(<1 \%)$ \\
$\quad$ Total combinational functions & $5 / 15,408(<1 \%)$ \\
Dedicated logic registers & $0 / 15,408(0 \%)$ \\
Total registers & 0 \\
Total pins & $50 / 347(14 \%)$ \\
Total virtual pins & 0 \\
Total memory bits & $0 / 516,096(0 \%)$ \\
Embedded Multiplier 9-bit elements & $0 / 112(0 \%)$ \\
Total PLLs & $0 / 4(0 \%)$ \\
\hline
\end{tabular}

Figure 4.8. Flow summary of 1 bit optimized ALU implemented on Intel /Altera DE0 (Quartus 2) 


\begin{tabular}{|c|c|c|c|c|c|c|}
\hline \multicolumn{7}{|c|}{ Propagation Delay } \\
\hline & Input Port & Output Port & $\mathrm{RR}$ & $\mathrm{RF}$ & $\mathrm{FR}$ & $\mathrm{FF}$ \\
\hline 1 & iA_ $X$ & oCout & 4,135 & 4,095 & 4,891 & 4,851 \\
\hline 2 & $\mathrm{iA} \_$X & 05um & 4,496 & 4.485 & 5,252 & 5,241 \\
\hline 3 & $\mathrm{BB}_{-}{ }^{\mathrm{Y}}$ & oCout & 4,125 & 4,085 & 4,858 & 4,818 \\
\hline 4 & BB_Y & 0Sum & 4,486 & 4.475 & 5,219 & 5,208 \\
\hline 5 & iCin & oCout & 4,104 & & & 4,868 \\
\hline 6 & iCin & 05um & 4.437 & 4.409 & 5.242 & 5,232 \\
\hline 7 & iso & oCout & 4,105 & 4,065 & 4,854 & 4,814 \\
\hline 8 & iso & 0Sum & 4.466 & 4.455 & 5,215 & 5,204 \\
\hline 9 & is1 & oCout & 4,226 & 4,186 & 4,968 & 4,928 \\
\hline 10 & iS1 & 05um & 4,587 & 4.576 & 5.329 & 5.318 \\
\hline 11 & is2 & oCout & 4,163 & 4,146 & 4,966 & 4,901 \\
\hline 12 & is2 & 05 um & 4.547 & 4.536 & 5.307 & 5,291 \\
\hline
\end{tabular}

Figure 4.9. Propagation Delay Summary of 1 bit ALU implemented on Intel /Altera DE0 (Quartus 2)

\begin{tabular}{||ll|}
\hline PowerPlay Power Analyzer Status & Successful - \\
Quartus II Version & 11.0 Build 208 07/03/2011 SP 1 SJ Web Edition \\
Revision Name & aluopt \\
Top-level Entity Name & aluopt \\
Family & Cyclone III \\
Device & EP3C16F484C6 \\
Power Models & Final \\
Total Thermal Power Dissipation & $103.99 \mathrm{~mW}$ \\
Core Dynamic Thermal Power Dissipation & $0.37 \mathrm{~mW}$ \\
Core Static Thermal Power Dissipation & $51.80 \mathrm{~mW}$ \\
I/O Thermal Power Dissipation & $51.82 \mathrm{~mW}$ \\
Power Estimation Confidence & Low: user provided insufficient toggle rate data \\
\hline
\end{tabular}

Figure 4.10. Power consumption summary of 1 bit ALU implemented on Intel /Altera DE0 (Quartus 2)

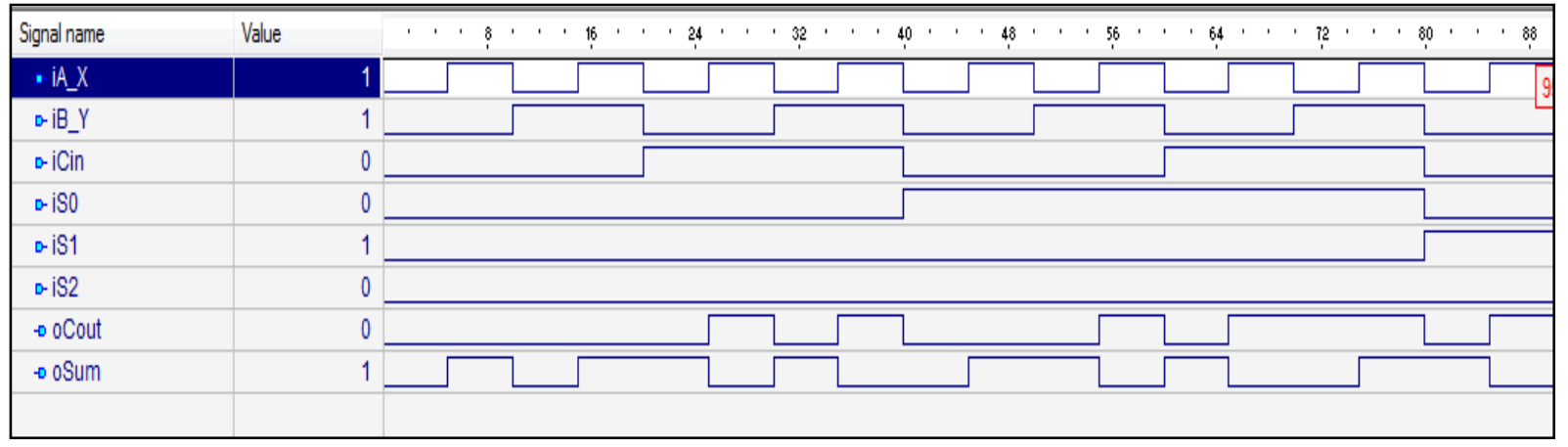

Figure 4.11. Output waveform of 1 bit optimized ALU 


\section{Optimized Approach Verification}

The comparison of flow summary (figure 2.2, $3.2 \& 4.8$ ) indicates that the number of elements required in implementation can been reduced in entity 'aluopt' comparison to entity 'alugen' and 'alugenopt' if higher order of ALU is being realized.

On observing the propagation delay report of entity 'alugen' (figure 2.3), 'alugen' (figure 3.3) \& entity 'aluopt' (figure 4.9), it was found that propagation delay in considerably less in 'aluopt' in comparison to 'alugen' and 'aluopt'. The highest propagation delay in 'alugen' is 6.12 nsec; in 'alugenopt' is $6.273 \mathrm{nsec}$ while in 'aluopt' is 8.270 nsec.

The power analyzer summary comparison (figure 2.4, $3.4 \& 4.10$ ) shows considerable power savings in 'aluopt' in comparison to 'alugen' can be obtained. The power readings obtained here depend on input test vector combination taken.

No benchmark work with similar formal specification of one-bit ALU is available. So, comparison of design strategy suggested in this paper cannot be done.

Table 5.1. Power comparison of ALU (Power in milliWatt)

\begin{tabular}{|c|c|c|c|}
\hline Entity Name & ALUgen & ALUGen opt & Aluopt \\
\hline $\begin{array}{c}\text { Total Thermal Power } \\
\text { Dissipation }\end{array}$ & 105.42 & 101.46 & 103.99 \\
\hline $\begin{array}{c}\text { Core Dynamic } \\
\text { Thermal Power } \\
\text { Dissipation }\end{array}$ & 0.76 & 0.63 & 0.37 \\
\hline $\begin{array}{c}\text { Core Static Thermal } \\
\text { Power Dissipation }\end{array}$ & 51.8 & 51.8 & 51.8 \\
\hline $\begin{array}{c}\text { I/O Thermal Power } \\
\text { Dissipation }\end{array}$ & 52.85 & 49.04 & 51.82 \\
\hline
\end{tabular}

\section{Conclusions}

The empirical measurements quantifying the gap between VLSI implementation of 'alugen', 'alugenopt' \& 'aluopt' has been presented in this paper.

In 'alugen' entity no concept of resource sharing had been used. Hence, for each corresponding arithmetic instruction execution in ALU block, one full adder block is required. On other hand in entities 'alugenopt' and 'aluopt' because of concept of resource sharing i.e. operator sharing and functionality sharing only one full adder block along with few multiplexer is required for implementation of arithmetic instruction in ALU.

Moreover, due to use of concept of optimized arithmetic expressions (viz. arranging expression trees for minimum delay, sharing common subexpression, merging cascaded adders with carry) along with resource sharing entity 'aluopt' is better in terms of area requirement in compare to entity 'alugenopt'.

On comparing the results obtained at frontend VLSI design, it is found that better saving in area or resource utilization, delay \& power can be obtained through the approach suggested in this paper.

Further, same approach if used for implementing higher order ALU can result in efficient realization at VLSI frontend level.

Further, optimization of full adder block using fast adder approach like carry look ahead adder, carry select adder, carry skip adder may achieve better results \& hence more better realization of ALU and hence Central Processing Unit (CPU) in any processor design ${ }^{[9,10,11]}$.

Also, the ALU design approach suggested in this paper if implemented at VLSI backend level may provide further optimized readings in terms of area, power \& delay ${ }^{[8,9 \& 10]}$.

Today, there are various tools like Aldec's Active HDL available in market \& few open sources like Deeds-DCS which diminishes the need to have thorough knowledge of HDL (Hardware Description Language). Just need herein is to have knowledge of digital circuits design.

The results and conclusions presented in this paper gives line of sight to beginners working in VLSI domain about the how to think about formal specification of any VLSI based project \& accordingly what methodology \& hence tool should be selected for design \& implementation.

\section{Conflicts of Interest}

The authors have no conflicts of interest to declare.

\section{REFERENCES}

[1] cseweb.ucsd.edu/ hepeng/cse143-w08/labs/ VHDL Referen ce / 09.pdf.

[2] Sabih Greez, "Algorithms for VLSI Design Automation", John Wiley \& Sons, Reprint 2000 edition, Clark T. Merkel, "A Matlab-Based Teaching Tool for Digital Logic", Mechanical Engineering, Rose-Hulman Institute of Technology, "Proceedings of the 2004 American Society for Engineering Education Annual Conference \& Exposition, American Society for Engineering Education, 2004.

[3] A.P. Godse, D.A Godse, "Digital Electronics", Third Revised Edition,2008.

[4] Greenfield, Joseph, "Practical Digital Design Using ICs", pub. by J. Wiley \& Sons.

[5] Jan M. Rabaey, Digital Integrated Circuits, Upper Saddle River, NJ: Prentice Hall, 1996.

[6] William Stallings, Computer Design and Architecture, Upper Saddle River, NJ:Prentice Hall, 1996.

[7] Douglas Perry, "VHDL Programming by Example", Fourth Edition, TataMcGraw-Hill, Eighth Reprint, 2006.

[8] N.H Weste, David Haris \& A.Banrjee, "CMOS VLSI Design, A Circuit \& System Perspective", Third Edition, Pearson Education.

[9] N.Ravindran, R.Mary Lourde, “ An Optimum VLSI Design 
Design of a 16-bit ALU", IEEE International Conference on Information \& Communication Technology Research (ICTRC), DOI: 10.1109/ICTRC.2015.7156419, Abu Dhabi, United Arab Emirates July, 2015.

[10] Prashant Gurjar, Rashmi Solanki \& Pooja Kansliwal, "VLSI Implementation of adders for high speed ALU”, IEEE Annual India Conference Indicon, Hyderabad, India, DOI: 10.1109/INDCON.2011.6139396, December, 2011.

[11] Sanjeev Sharma, "FPGA Implementation of 1-bit ALU", International Conference on Information, Communication and Embedded System(ICICES), Chennai, India, 25-26 February, 2012. 